Voix et Images

volxetimages

\title{
Échos et « survenances " dans les versions médiatiques du
} Survenant

\section{Echoes and "Survenances" in Media Versions of Le Survenant Ecos y "surveniencias" en las versiones mediáticas de Le Survenant}

\section{Danielle Aubry}

Volume 33, numéro 3 (99), printemps-été 2008

Germaine Guèvremont. Nouvelles survenances

URI : https://id.erudit.org/iderudit/018671ar

DOI : https://doi.org/10.7202/018671ar

Aller au sommaire du numéro

\section{Éditeur(s)}

Université du Québec à Montréal

\section{ISSN}

0318-9201 (imprimé)

1705-933X (numérique)

Découvrir la revue

Citer cet article

Aubry, D. (2008). Échos et « survenances » dans les versions médiatiques du Survenant. Voix et Images, 33(3), 41-53. https://doi.org/10.7202/018671ar

\section{Résumé de l'article}

Lorsqu'une oeuvre romanesque est adaptée pour un ou plusieurs autres médias, la question de la fidélité au texte original surgit inévitablement, tel un réflexe, qui élude les particularités esthétiques et matérielles du médium d'arrivée. Or, la transposition du Survenant pour la radio et la télévision a exigé une expansion considérable du récit en épisodes distincts s'étalant sur une période souvent très longue, ce qui a demandé de la part de l'auteure de créer de toutes pièces de larges pans d'intrigues, qui ne figuraient pas dans le roman ou y étaient à peine ébauchés. Par ailleurs, chaque médium imposant des contraintes techniques précises, Guèvremont, qui en avait pris la pleine mesure, a souvent utilisé celles-ci de façon inventive. Sur ce plan, si les passages médiatiques nous font perdre en partie la beauté stylistique du roman, les dialogues, riches des inflexions que leur donnent les comédiens, contribuent à instaurer une poésie unique en son genre. 


\title{
ÉCHOS et "SURVENANCES"DANS \\ LES VERSIONS MÉDIATIQUES DU SURVENANT
}

\author{
$+++$ \\ DANIELLE AUBRY† \\ Université du Québec à Montréal
}

L'immensité du corpus des séries de fiction radiophoniques et télévisuelles québécoises ainsi que la question épineuse des archives ont de quoi rebuter la plupart des critiques littéraires, et à l'exception de Renée Legris et de Pierre Pagé, ils sont peu nombreux à s'être aventurés sur ce terrain encore largement en friche. Yvan G. Lepage fait d'ailleurs remarquer à ce sujet, dans son ouvrage consacré à Germaine Guèvremont en 1998, qu'il n'a pas abordé ses œuvres médiatiques, d'une part parce qu'elles n'étaient disponibles que sur microfilm, «ce qui en rend[ait] la consultation malaisée» et d'autre part parce que ces textes dramatiques, "hors du cadre pour lequel ils ont été conçus, et sans la voix ou la présence des interprètes qui leur ont donné vie, [...] pâlissent et perdent un peu de leur vertu ${ }^{1}$ ». Le critique évoque dans ce passage la disparition presque totale des émissions radiophoniques et télévisuelles dont il ne reste, à part quelques épisodes des trois téléromans, que les scénarii dactylographiés ${ }^{2}$.

À cet égard, s'il est tout à fait justifié de craindre la lecture de milliers de pages de textes privés de leur texture sonore ou audiovisuelle, il est tout de même nécessaire que l'on s'y intéresse puisqu'ils représentent, quantitativement, la part la plus importante de l'œuvre de Guèvremont. En effet, dès le moment où l'auteure a commencé à écrire des séries pour la radio, en 1953, puis pour la télévision, en 1954, elle a abandonné tout autre projet littéraire et journalistique, faute de temps, car la quantité de textes à écrire ne lui laissait que très peu de loisirs pour d'autres activités créatives. Mais ce qu'il serait loisible de considérer comme une rupture avec le monde des lettres pourrait aussi être interprété comme une continuité; ainsi que l'ont démontré les travaux de Renée Legris ${ }^{3}$, le texte médiatique possède un fondement littéraire et peut donc être appréhendé selon des outils théoriques développés

$$
++
$$

1 Yvan G. Lepage, Germaine Guèvremont. La tentation autobiographique, Ottawa, Les Presses de l'Université d'Ottawa, coll. "EEuvres et auteurs», 1998, p. 198. 2 Consulter, au sujet des archives radiophoniques, l'introduction de l'ouvrage de Pierre Pagé, Répertoire des œeuvres de la littérature radiophonique québécoise, 1930-1970, Montréal, Fides, coll. "Archives québécoises de la radio et de la télévision», 1975, p. 19-63. 3 Voir, notamment, Robert Choquette, romancier et dramaturge de la radio-télévision, Montréal, Fides, coll. "Archives québécoises de la radio et de la télévision", 1977, 287 p. 
en fonction de la littérature. En outre, les versions médiatiques du Survenant constituent des adaptations de ses deux romans, Le Survenant (1945) et Marie-Didace ${ }^{4}$ (1947), qui ont marqué l'histoire littéraire du Québec en «introduisant dans un univers traditionnellement étriqué d'hommes asservis à la terre, un héros charismatique, chantre de l'insouciance, de la jeunesse et de la liberté ${ }^{5}$ ». Par ailleurs, ces transpositions donnent non seulement un second souffle à l'œuvre littéraire en la rendant accessible à un public élargi, mais elles lui apportent également de nouvelles dimensions, tant par l'élargissement considérable du récit que par les traits distinctifs des médias eux-mêmes.

Sur ce plan, si l'on veut évaluer de façon adéquate le corpus médiatique, il convient de l'envisager non comme un appauvrissement par rapport à la forme romanesque dont il dérive, mais comme un ensemble d'œuvres à part entière renfermant des qualités propres aux médias qui leur ont donné vie. Ainsi, le critique doit se départir d'une posture esthétique souvent implicite fondée sur la "fidélité » à l'œuvre originale et sur la littérarité, posture qui occulte la puissance d'expression singulière des médias d'arrivée. À ce chapitre, un autre critère esthétique lié au premier entrave souvent la compréhension des œuvres médiatiques: l'unicité du texte littéraire que l'on pourrait définir comme l'interrelation de toutes les composantes de l'œuvre qui met en lumière une convergence sémantique empêchant l'expansion infinie du sens. C'est cette notion, soutenue notamment par l'idée que l'intention auctoriale devient l'arbitre suprême du sens, qui conférerait au texte sa légitimité, sa valeur artistique.

Or, un tel endiguement perd une bonne partie de sa validité pour qui étudie des textes aux multiples intrigues arborescentes d'une grande étendue, réseau textuel occupant un espace multimédiatique complexe qui empêche une lecture linéaire des œuvres. Dans cette perspective, le roman n'est plus le seul point de fuite à partir duquel les œuvres dérivées sont jugées: le critique doit aussi tenir compte non seulement du caractère distinctif de chaque médium, mais aussi des genres médiatiques auxquels l'œuvre appartient, en l'occurrence le radioroman et le téléroman. Lorsque Guèvremont entreprend la version télévisée du Survenant, le radioroman existe depuis une vingtaine d'années. Robert Choquette, l'initiateur du genre, avait consacré des efforts soutenus pour mettre au point des techniques $\mathrm{d}^{\prime}$ « écriture sonore ${ }^{6}$ » adaptées au médium et à son mode de diffusion parcellaire. Ce savoir-faire, qu'incarnent à merveille La pension Velder (1938-1942) et Métropole (1943-1956) diffusés par CBF, la même station qui mettra en ondes Le Survenant, a donc instauré une tradition médiatique incontournable pour Germaine Guèvremont. De même, au moment où la romancière s'engage dans l'écriture de la version télévisée du Survenant, elle bénéficie non seulement de son expérience récente comme

$$
+++
$$

4 Dans une moindre mesure, dans ses contes recueillis dans En pleine terre. Paysanneries. Trois contes (Montréal, Paysana, 1942, 159 p.) on voit apparaître certains des personnages qui seront repris dans les romans, puis dans leurs adaptations médiatiques. 5 Yvon G. Lepage, op. cit., p. 8. À noter que même si le Survenant est absent de Marie-Didace, il hante le roman dans la mémoire des personnages dont il a traversé la vie. $\mathbf{6}$ L'expression appartient à Renée Legris, "De la mémoire collective à l'histoire du thêâtre médiatique», L'Annuaire thêâtral, nos 5-6, automne 1988/printemps 1989, p. 105. 
auteur radiophonique, mais peut aussi prendre modèle sur le téléroman La famille Plouffe de Roger Lemelin, en ondes depuis une saison, et dont la formule est devenue le prototype essentiel du genre téléromanesque ${ }^{7}$.

La démarche utilisée pour étudier l'œuvre médiatique de Guèvremont est donc principalement fondée sur le travail créateur qui préside à la matérialisation des œuvres radiophoniques et télévisuelles, processus qui ne peut échapper à une compréhension préalable des médias, à la reconnaissance de leurs codes communs et singuliers. Dans cette optique, je m'appuie sur le principe de rhétorique ${ }^{8}$, que j'ai déjà développé dans mes travaux antérieurs puisqu'il me permet de circonscrire la dimension performative du scénario, étape transitoire vers une production audio ou audiovisuelle de fiction. Cette performance, obéissant à un impératif que partagent les médias électroniques, celui d'atteindre le plus grand public possible, repose sur l'emploi (créatif ou stéréotypé) d'un ensemble de conventions narratives et dramatiques déterminées par la sérialisation des textes et dont plusieurs remontent au XIX siècle.

\section{RHÉTORIQUE INTERMÉDIALE DU TEXTE MÉDIATIQUE}

Le croisement entre une certaine tradition littéraire et les particularités techniques, commerciales et esthétiques de la radio et de la télévision, nées à une trentaine d'années d'intervalle, a donné lieu à des pratiques intermédiales précises qui assurent l'intelligibilité du texte sériel. Cette intelligibilité, sur le plan formel, est généralement marquée par une démarche mimétique empruntée au roman, un réalisme psychologique et social conditionné par la vie domestique, d'où la résurgence d'une modalité feuilletonesque typique, celle de la chronique familiale, que l'on pourrait qualifier de "fresque intime», pour reprendre l'expression du chroniqueur Jean Hamelin ${ }^{9}$. Par ailleurs, le dispositif technique des médias électroniques impliquant la diffusion de chaque émission selon une durée fixe et un déroulement inexorable, le texte narratif devient un spectacle qui emprunte quelques-unes de ses conventions au théâtre. Outre le découpage en unités dramatiques (actes, scènes) qui le structurent, c'est le recours systématique aux dialogues qui forme le véhicule le plus important de transmission du récit, qu'il soit radiophonique ou télévisuel. Comme l'avaient bien compris Choquette et, à sa suite, Guèvremont, le discours direct exige une attention soutenue au langage, et cela est d'autant plus important ici que l'auditoire du radioroman est étendu et composite. Les auteurs médiatiques apportent donc un soin particulier à l'élaboration d'un langage qui stylise des parlers et des thèmes accessibles, qui se rapprochent de la vie urbaine ou rurale de l'époque, dans

$$
++
$$

7 À noter que cette série avait préalablement pris la forme d'un radioroman diffusé aussi sur CBF de 1952 à 1955. Les versions radiophonique et télévisuelle se sont donc chevauchées pendant trois ans. $\mathbf{8}$ À ce propos, voir Danielle Aubry, Du roman-feuilleton à la série télévisuelle. Pour une rhétorique du genre et de la sérialité, Berne, Peter Lang, 2006, 244 p. 9 "Reparlons donc (en bien toujours) du Survenant», chronique "Vu et entendu ", Le Petit Journal, 9 décembre 1956, p. 96 
toute l'épaisseur de sa quotidienneté. Ainsi, de «l'obscurité optique ${ }^{10}$ » du texte radiophonique naissent pourtant des images dont la familiarité préfigure déjà la matérialité visuelle du téléroman.

Dans cette perspective, le langage est un vecteur essentiel du réalisme, car il contribue à la caractérisation des personnages dont il désigne l'appartenance ethnique et sociale, le niveau d'éducation, les idiosyncrasies qui se manifestent notamment par des phrases récurrentes. Ainsi, dans les versions médiatiques du Survenant, on trouvera par exemple des expressions populaires telles que «C'est pas franc» de Phonsine et «Il est pas de rien» de Didace ou les termes en latin qu'affectionne le docteur Desgroseillers. En ce sens, le langage élaboré par Guèvremont permet aussi d'établir des clivages sociaux entre les personnages, clivages favorisant l'éclosion de conflits qui ne sont pas seulement d'ordre psychologique, comme dans le roman, mais qui explorent des questions sociales modernes, dont l'aspiration à progresser dans l'échelle sociale, le désir d'une vie meilleure, ou tout simplement différente, plus exaltante, l'envie de gagner sa vie autrement que sur une terre. Ainsi, la langue du terroir, même si elle domine toujours dans les récits médiatiques, se trouve souvent confrontée à des parlers urbains ou carrément à l'anglais, langue associée aux affaires ${ }^{11}$. Ajoutons que l'effet de réel créé par le langage est également favorisé par la voix unique du comédien qui le matérialise, grâce au timbre, au rythme, à l'intensité, à l'étoffe, à l'intonation et à l'accent, qui lui sont propres. À la radio, tout particulièrement, la voix constitue un ancrage sémantique essentiel car, portant un ensemble de marques récurrentes, elle devient familière à l'auditeur, qui construit de la sorte une mémoire auditive le guidant dans les méandres narratifs du feuilleton. Et lorsqu'un personnage est interprété par le même acteur dans toutes les versions médiatiques d'un roman (je pense aussi au cinéma), sa voix, son visage, sa gestuelle infléchissent la lecture ou relecture de l'œuvre littéraire. Plus largement, il m'apparaît évident que celle-ci ne peut plus être envisagée comme une entité parfaitement autonome, mais qu'elle fait partie d'un agrégat textuel multimédiatique dont le sens émerge "dans sa totalité, pas d'une façon linéaire, mais synergique ${ }^{12}$ ».

Sur le plan structurel, le feuilleton radiophonique et le téléroman ont cela de commun avec le feuilleton littéraire qu'ils se composent de séries d'unités dont l'expansion narrative est potentiellement infinie. À ce propos, il convient de distinguer les œuvres uniques (radiothéâtre et téléthéâtre) des œuvres sérielles, qui ne peuvent être appréhendées que dans la juxtaposition de syntagmes narratifs se déroulant selon une continuité à la fois temporelle et diégétique plus ou moins longue.

10 Jürgen E. Müller, «Texte et médialité, une introduction», Texte et médialité, Mannheim, Lehrstuhl Romanistik I, coll. «Mannheim-Analytiques», 1987, 417 p. 11 Pour plus de précisions à ce sujet, consulter le chapitre II du mémoire de maîtrise de James Herlan, «Le Survenant de Germaine Guèvremont. Une étude comparative du roman et du radio roman », Trois-Rivières, Université du Québec à Trois-Rivières, 1980, p. 31-48. Voir aussi James Harlan, «L'adaptation radiophonique du Survenant. Structure dramatique », Essays on Canadian Writing, nº 15, été 1979, p. 69-85. 12 Heidy Peeters, « Mediatic Synergy. Towards a Networked Conception of Adaptation", article à paraître, élaboré à partir de sa communication au colloque Adaptation(s). Transferts et société organisé par le Centre de recherche sur l'intermédialité, sous la direction de Michael Eberle-Sinatra, Danielle Aubry et al., du 15 au 17 novembre 2006. 
Bien que les œuvres uniques partagent un certain nombre de procédés d'écriture avec le feuilleton, la forme feuilletonesque impose des contraintes supplémentaires à l'auteur. Celui-ci doit en effet, en premier lieu, concevoir maintes intrigues parallèles se rencontrant à l'occasion sous la gouverne d'un motif narratif dominant. Ensuite, malgré la variété de ces intrigues, il lui faut en établir la continuité, la logique narrative, tout en tenant compte des interruptions publicitaires et de l'hiatus séparant la diffusion de chaque épisode. Dans ce régime de récit perforé, afin d'instaurer une certaine cohérence structurelle, le feuilletoniste a recours à des séries de «combinaisons répétitives ${ }^{13}$ ", des variations sur un même thème tirées d'un répertoire de motifs et de situations dramatiques préalablement exploités par la littérature (et, par la suite, au cinéma) et assez familières au public pour qu'il puisse en saisir aussi les écarts. Ainsi, l'élément déclencheur de la version romanesque du Survenant, la venue d'un étranger dans une communauté fermée sur elle-même, trope narratif très répandu, s'accompagne d'autres motifs tout aussi courants : l'identité mystérieuse du Survenant; la déception d'un père face à l'ineptie de son fils et la hantise de la continuité de sa lignée; la rivalité entre deux frères (on peut considérer Amable et le Survenant comme deux frères puisque ce dernier représente le fils que Didace a toujours rêvé d'avoir); les conflits entre voisins, Didace et Pierre-Côme, nourris par les ambitions et la vanité de ce dernier; l'amour déçu d'Angélina; la menace de l'arrivée d'une belle-mère dans la vie des Beauchemin, etc.

Les personnages récurrents (ou réapparaissant) constituent un autre trait essentiel de l'intelligibilité médiatique en créant des instances de cohésion additionnelles. Dans toutes les versions du Survenant, le protagoniste demeure l'inconnu, lequel, par sa présence, «agit [...] à la façon d'un catalyseur qui tout en faisant progresser l'action, permet d'étudier les personnages en présence ${ }^{14} »$. Agent primordial de l'action, le Survenant continue de jouer le rôle, qui lui était dévolu dans le roman, de révélateur psychologique avec, comme principaux interlocuteurs, Didace, Phonsine, Amable et Angélina. Toutefois, la narrativité feuilletonesque exige, pour se déployer dans la durée, l'emploi d'un nombre élevé de personnages qui, de secondaires ou épisodiques dans le roman, prennent plus d'ampleur dans les textes sérialisés. Ainsi, les familles Salvail et Provençal, particulièrement les jeunes, font l'objet de développements narratifs importants qui servent surtout à développer les motifs sentimentaux de la trame narrative. Le personnage de Catherine Provençal, par exemple, à peine évoqué dans le roman, devient un personnage à part entière dans le radioroman, se révoltant contre sa condition de vieille fille et de servante de sa famille et nourrissant, comme bien d'autres jeunes filles de son entourage, son désir d'évasion à travers le Survenant. L'amplification touche aussi un autre personnage épisodique du roman, Beau-Blanc, un illettré du village qui, comme le souligne si justement James Herlan, "reflète une image défigurée et grotesque du Survenant ${ }^{15}$ ». L'une des richesses du texte sériel tient justement à la possibilité de

13 Renée Legris, Robert Choquette, romancier dramatique de la radio télévision, op. cit., p. 52.14 Rita Leclerc, Germaine Guèvremont, Montréal/Paris, Fides, coll. «Écrivains canadiens d'aujourd’hui », 1963, p. 40.15 James Herlan, op. cit., p. 67. 
multiplier ce type de réfraction actantielle, non seulement sur un mode contrasté, mais aussi homologique, comme c'est le cas avec Joinville, incarnation plus juvénile du Survenant. Les contradictions du protagoniste, déchiré entre son irrésistible goût de la liberté et un désir inavoué de stabilité, se trouvent de la sorte extériorisées grâce à l'existence d'adjuvants et de doubles dérisoires ${ }^{16}$.

Par ailleurs, le développement de personnages secondaires qui s'allient à la cause des antagonistes du Survenant augmente l'intensité des conflits qui l'opposent à ceux qui ne veulent pas de lui au Chenal du Moine. Il en va ainsi d'Odilon, fils de Pierre-Côme Provençal, succédané du père dont il endosse la haine de l'étranger, haine exacerbée par la jalousie puisqu'il convoite Angélina (ou plutôt ses biens) qui, elle, n'a d'yeux que pour le Survenant. Quant à l'invention de nouveaux personnages contribuant à la prolifération actantielle si caractéristique du feuilleton, elle permet aussi d'ouvrir l'univers du Chenal à des sphères sociales plus diversifiées. À ce titre, le docteur Sévère Desgroseillers, loin de ne s'occuper que des maux physiques de ses clients, profite de sa situation professionnelle pour faire de la politique. Dans la foulée de ses activités comme chef de l'aile régionale d'un parti qui n'est pas nommé, il devient le centre de questions et de comportements qui appartiennent à l'espace public.

Le nombre accru de personnages risquait de devenir source de confusion pour le public; Guèvremont, suivant en cela une tradition déjà classique à la radio, donne à la plupart d'entre eux un "mot de chevet ${ }^{17}{ }^{\prime}$. Si, dans son roman, l'auteur utilise parcimonieusement certaines expressions comme le fameux «Neveurmagne» du Survenant et le «Journée d'la vie!» de Phonsine, elle les multiplie dans les versions médiatiques. Ainsi, non seulement Phonsine et le Survenant émaillent-ils leurs répliques de l'expression qui leur avait été attribuée dans le roman, mais encore presque chaque personnage possède son "patois» (terme que l'on utilise dans le milieu des scénaristes de la télévision): à Amable est associé "Ça vient de s'éteindre!»; à Didace, «Cré race de monde!» etc. Qui plus est, les personnages répètent ce patois si fréquemment que cette forme de ponctuation identitaire prend forcément une dimension comique ${ }^{18}$.

En dernier lieu, l'accessibilité de l'œuvre médiatique dépend dans une large mesure d'un agent cohésif résolument intermédial, le genre. D'ailleurs, les appellations hybrides qui sont apparues, dès l'émergence de chaque médium, pour désigner certaines séries de fiction ont d'emblée reflété l'association entre un genre établi et le médium émergent: radioroman, radiothêâtre, feuilleton radiophonique, feuilleton dramatique, téléspectacle, téléroman, téléthêâtre sont autant de mots composites qui témoignent d'une intense activité intermédiatique. Mais ces appellations ne sauraient à elles seules représenter la complexité de la rhétorique du genre à l'œuvre dans chaque radioroman ou téléroman.

16 Ibid., p. 65. 17 On se souviendra du «viande à chien» de Séraphin, l'avare d'Un homme et son péché de Claude-Henri Grignon. 18 L'usage systématique du patois, lancé à la radio, se transmettant par la suite à un nombre élevé de téléromans pionniers, a été perpétué par les générations successives d'auteurs, tels Guy Fournier et Victor-Lévy Beaulieu. Bien que Fournier l'exploite surtout sur le mode de la comédie, chez Beaulieu, en revanche, le patois se teinte d'un certain maniérisme qui, au diapason du langage très stylisé de cet auteur, brise l'effet de réel, la fausse transparence recherchée par la plupart des auteurs de séries médiatiques. 
Le Survenant, roman rural à caractère historique, comporte aussi les éléments d'une chronique familiale narrée dans une tonalité dramatique (voire tragique), allégée, à l'occasion, grâce à quelques effets comiques. Dans les transpositions médiatiques, par contre, le registre comique prend une importance nettement plus grande que dans le roman, ce qu'alimente la place accrue accordée aux personnages mineurs du roman, lesquels possèdent presque tous un aspect caricatural, chargé, comme Beau-Blanc, Bedette, Jacob, etc. ${ }^{19}$. La prédominance du comique, si elle est occasionnée en partie par l'expansion du récit, est également tributaire de sa vocation commerciale, de la nécessité de plaire à un auditoire très diversifié, mais surtout populaire, qui n'a pas nécessairement eu l'occasion de lire le roman. Mais, pour rendre justice au talent de Germaine Guèvremont, il faut mentionner son sens de l'observation et la perspicacité de sa peinture satirique des comportements contradictoires de ses personnages. En témoigne une intrigue extrêmement drôle figurant dans la version télévisée, qui relate la sempiternelle réélection de Pierre-Côme comme maire du Chenal du Moine. Jacob explique ainsi le conservatisme de ses pairs:

Ceux qui étaient pour lui ont voté contre lui pour lui donner une bonne leçon, pour pas qu'il arrive trop fort. (STOP). Ceux qui étaient contre ont voté pour, pendant [sic, pensant] que ça tirerait pas à conséquence. (STOP) Comme y avait plus de contre que de pour, (STOP) il s'est trouvé élu. (STOP) C'est clair ${ }^{20}$.

À des degrés divers, sous une forme plus ou moins atténuée, le mode mélodramatique traverse également les œuvres médiatiques, se manifestant, sur le plan de l'intrigue, par des motifs typiques du mélodrame, notamment la victime innocente torturée moralement (et parfois, physiquement) par un bourreau qui représente souvent une figure parentale tordue. L'exemple le plus connu est sans doute celui d'Aurore, mais la relation malheureuse qu'entretient Donalda avec son mari Séraphin ne le lui cède en rien. Si, dans l'ensemble des textes du Survenant, la victime demeure essentiellement personnifiée par Angélina ${ }^{21}$, que le Grand-dieudes-routes fait languir d'amour, la tonalité du roman est plus tragique que mélodramatique. Toutefois, comme, dans les versions sérielles, cette victimisation s'inscrit dans la durée, elle produit un double effet contradictoire. D'abord, la souffrance, se perpétuant en d'infinies variations sérielles, semble s'alourdir de cette accumulation,

19 Renée Legris constate, en parlant de La pension Velder de Robert Choquette, que dans la version novellisée du radioroman, Élise Velder, "l'auteur a éliminé toute la dimension comique de son œuvre radiophonique» (Robert Choquette, romancier dramatique de la radio télévision, op. cit., p. 35). Même si le processus d'adaptation est ici inversé par rapport au Survenant, le fait est que l'humour occupe une place prépondérante dans les versions radiophoniques par rapport au roman; soulignons que c'est aussi le cas d'Un homme et son péché de Claude-Henri Grignon. 20 Germaine Guèvremont, Le Survenant, Téléroman (CBFT, 30 novembre 1954 au 9 juillet 1957), Bibliothèque et Archives Canada, Fonds Germaine Guèvremont, LMS 0260, 2004-03, série 2, $\mathrm{n}^{\circ} 10,1^{\text {er }}$ février 1955, p. 2. Désormais, les références à ce document seront placées entre parenthèses dans le texte et indiquées par le sigle TRS, suivi du numéro d'épisode, de la date et du folio. 21 Et, dans une moindre mesure, par Phonsine, terrorisée par un beau-père tyrannique et obligée de protéger son mari Amable, aussi vil que vellêitaire, de ses foudres. 
prendre la couleur de la fatalité, phénomène particulièrement sensible dans le radioroman, dans lequel le Survenant se montre plus insouciant et volage. Toutefois, l'entrelacement d'autres intrigues, dont certaines sont comiques, provoque un décentrement de ce schéma d'autant plus manifeste qu'il se réfracte en d'autres "victimes » tombées sous les charmes du Survenant: Bedette, Catherine, Rose-de-Lima et même Phonsine. Cette dispersion donne au récit sériel (surtout au radioroman) un caractère empreint de relativité, contingence qu'accroît l'absence de dénouement.

L'un des tropes narratifs les plus répandus du genre est fondé sur le mystère, surtout quand celui-ci concerne l'identité. Les deux versions médiatiques du Survenant font mousser cet aspect, comme l'illustre l'intrigue axée sur le mystérieux Eric A. MacDonald, que recherche activement un journaliste, Albert Lapensée, et qui serait en réalité nul autre que le Survenant. L'obsession des origines du Survenant, qui hante déjà Didace dans le roman, se conjugue dans les œuvres sérialisées à l'amour d'Angélina, la "monopathie» respective des personnages s'unissant dans divers complots pour empêcher son départ. Les dialogues peuvent aussi, de façon plus minimaliste et ponctuelle, comporter une certaine surcharge mélodramatique. Ainsi, lorsque le Survenant demande à Angélina pourquoi Catherine a seule la charge de la maisonnée:

$\begin{array}{ll}\text { ANGELINA } & \text { Ben... Un peu gênée. Vous devriez le savoir ! } \\ \text { SURVENANT } & \text { Savoir... quoi? } \\ \text { ANGELINA } & \text { Sa mère... est enfermée. } \\ \text { SURVENANT } & \text { Enfermée... où? } \\ \text { ANGELINA } & \text { Parlez pas si fort! Quéqu'un pourrait nous entendre. } \\ \text { SURVENANT } & \text { Est-ce qu'elle aurait commis... un crime? } \\ \text { ANGELINA } & \text { Voyons! Y avait pas d'meilleure femme au monde. Bas. Est enfer- } \\ & \text { mée... à l'âsile } 22 \text { ! }\end{array}$

Dans ce passage sont réunis plusieurs traits typiquement mélodramatiques: sur le plan de la rhétorique, l'insistance sur le mode interrogatif et la suspension, marques du discours représentant le mystère, puis l'exclamation finale, qui transmet la révélation (surprise, coup de thêâtre); sur le plan des stratégies narratives, l'allusion à des motifs sensationnalistes tels que l'enfermement (associé implicitement, au début du passage, à la prison), le crime et la folie, tous exploités à l'envi dans les feuilletons littéraires. Cette révélation va d'ailleurs servir à nourrir le dénouement de l'épisode qui se clôt avec fracas lorsque Didace, semonçant son rival de toujours, Pierre-Côme, au sujet de sa fille Catherine, lui déclare que s'il continue à empêcher sa fille de sortir de la maison, elle «va aller échouer là où c'est qu'est ta femme!», c'est-à-dire à l'asile. D'autres procédés empruntés au mélodrame et dont les

22 Germaine Guèvremont, Le Survenant, Radioroman (CBF, 31 août 1953 au 6 mai 1955; repris à CKVL, septembre 1962 à juin 1965), Bibliothèque et Archives Canada, Fonds Germaine Guèvremont, LMS 0260, 200403, série 1, nº 61, 17 décembre 1962, p. 5. Désormais, les références à ce document seront placées entre parenthèses dans le texte et indiquées par le sigle $R R S$, suivi du numéro d'épisode, de la date et du folio. 
feuilletonistes ont par la suite fait un usage parfois abusif jouent un rôle privilégié dans les dramatiques sérielles. Ainsi, le rebondissement, le coup de thêâtre, la surprise, le suspense, l'anticipation et le monologue pathétique, dont savait si bien jouer Alexandre Dumas, réapparaissent dans les œuvres médiatiques avec plus ou moins de bonheur, selon le talent des feuilletonistes. Bien que ces procédés aient d'abord été employés dans le contexte d'œuvres uniques, ils ponctuent efficacement une matière narrative fractionnée en unités scéniques et en interruptions plus ou moins longues séparant la diffusion des épisodes. On ne s'étonnera donc pas que l'auteur, afin de maintenir l'attention du public, s'applique à galvaniser le récit par des moyens qui ont fait leurs preuves au thêâtre et dans la littérature. Ainsi, le narrateur du Survenant termine-t-il un épisode radiophonique en évoquant à mots couverts l'alcoolisme du héros éponyme: «Le Survenant commencerait-il à se montrer sous son vrai jour? À demain.» (RRS, no 58, 12 décembre 1962, p. 6) Le suspense et l'apostrophe temporelle établissent un pont qui a pour but de combler le hiatus creusé entre chaque diffusion en attisant la curiosité de l'auditoire.

\section{RHÉTORIQUE STRUCTURALE DU FEUILLETON RADIOPHONIQUE}

Quoique les principes de cohérence du radioroman et du téléroman comportent un certain nombre de points communs, ils diffèrent en revanche sur d'autres aspects, différences attribuables à certaines caractéristiques spécifiques de chaque médium. L'une des distinctions les plus évidentes entre les deux genres est reliée au type de sérialisation dont ils font l'objet. Ainsi, le radioroman, qui est diffusé quotidiennement, se modèle plus strictement sur son ancêtre, le feuilleton romanesque, grâce à la prolifération d'intrigues parallèles portées par un nombre important de personnages. Pour définir ce foisonnement narratif, Renée Legris utilise le terme de «cycles dramatiques ${ }^{23}$ ", utile à plus d'un titre. D'abord, il permet d'envisager les intrigues selon la dynamique propre à tout feuilleton, celle de la répétition et de la variation. Ensuite, il désigne un autre de ses traits, typiquement feuilletonesque: sa sérialité ouverte, grâce à laquelle il devient possible de repousser indéfiniment une fin ultime. À cet égard, on peut constater d'emblée un écart fondamental entre Le Survenant littéraire et sa version radiophonique. L'intrigue du roman, qui se concentre sur les effets de la venue d'un étranger sur les habitants d'une petite communauté rurale, est linéaire et tend vers un dénouement inévitable.

Dans le radioroman, l'action se ramifie considérablement et se développe selon les quatre modalités suivantes : intrigues s'inspirant du roman, mais très détaillées dans des scènes qui n'existent pas dans le roman; intrigues inédites qui forment des variations à partir du grand axe thématique du roman, à savoir la fascination ou l'aversion (souvent un mélange des deux) suscitée par le Survenant; ajout d'intrigues totalement inédites, surtout liées à des personnages secondaires à peine

$$
++
$$


évoqués dans le roman, et qui occupent un espace narratif plus considérable dans le radioroman; dialogues thématiques qui n'ont pas de fonction narrative précise mais qui brodent à partir de la caractérisation des personnages ou qui commentent l'action.

Toutes ces extensions narratives appartiennent à des registres dramatiques qui n'existaient pas dans le roman, ou du moins qui y apparaissaient de façon très subtile ou larvée: le mélodrame et la comédie. Par ailleurs, une partie d'entre elles s'apparentent à une forme de dilution bien connue des feuilletonistes littéraires, ces «interminables conversations » dont parle Eco au sujet de certains romans de Dumas ${ }^{24}$. Ce procédé d'amplification, diamétralement opposé à la concentration émotive du mélodrame, s'y associe pourtant dans la grande majorité des œuvres sérielles, association paradoxale qui est devenue une constante esthétique dans leurs diverses manifestations médiatiques.

La rhétorique de l'amplification résulte à l'évidence des pressions mercantiles du médium sur le plan de la productivité, qui oblige l'auteur à écrire une grande quantité de textes (équivalant à $1 \mathrm{~h} 20$ de durée utile hebdomadaire par rapport à 30 minutes pour le téléroman) tout en respectant des échéances très serrées. Il n'est donc pas étonnant de constater la fréquence de scènes qui n'ont aucune fonction narrative précise et dans lesquelles le dialogue devient une sorte de commentaire d'une action ou d'un motif psychologique. Bien sûr, comme le fait valoir James Herlan ${ }^{25}$, les contraintes de l'écriture radiophonique obligent l'auteur à cette amplification, dont on pourrait trouver mille exemples dans Le Survenant radiophonique; il en va ainsi d'une conversation entre Amable, le fils de Didace, et sa femme Phonsine:

\begin{tabular}{|c|c|}
\hline NARRATEUR & $\begin{array}{l}\text { Si Pierre-Côme trouve qu'Amable fait preuve de perspicacité envers } \\
\text { le Survenant, il ne peut tout de même pas vanter sa vaillance... }\end{array}$ \\
\hline AMABLE & Il bâille. \\
\hline PHONSINE & Tu t'endors pas déjà, Amable? \\
\hline AMABLE & $\begin{array}{l}\text { Je tombe de sommeil. La grand'air, y a rien de pire! Un vrai tue- } \\
\text { monde! }\end{array}$ \\
\hline PHONSINE & $\begin{array}{l}\text { Journée de la vie, si tu continues, tu vas finir par attraper la maladie } \\
\text { du sommeil. }\end{array}$ \\
\hline AMABLE & Il bâille. Je pense que je veillerai pas tard. \\
\hline PHONSINE & $\begin{array}{l}\text { Rien qu'à te voir bâiller, c'est assez pour donner l'endormitoire à je } \\
\text { sais pas qui. }\end{array}$ \\
\hline AMABLE & J'vas me coucher. \\
\hline PHONSINE & T'as pas peur de faire des plaies de lit, toujours couché de même? \\
\hline AMABLE & $\begin{array}{l}\text { C'est pas de ma faute si je m'endors à rien }[\ldots] . \text { ( } R R S, n^{0} 59,13 \text { dé- } \\
\text { cembre } 1962, \text { p. 5) }\end{array}$ \\
\hline
\end{tabular}

24 Umberto Eco, De Superman au surhomme, traduit de l'italien par Myriem Bouzaher, Paris, Grasset, 1993, p. 101. 25 James Herlan, op. cit., p. 22-23. 
Ce passage brode sur la paresse proverbiale d'Amable, trait de caractère qui avait été établi dans le roman, mais jamais de façon aussi explicite. Bien que le dialogue télévisuel puisse aussi tomber parfois dans la ratiocination, ce procédé d'extériorisation des pensées des personnages, qui prend plutôt la forme du style indirect libre et se fait beaucoup plus discret dans le roman, est tout à fait propre au radioroman. Car, tout en relevant du principe de dilution qui permet d'allonger les textes, il reflète surtout la nature orale du médium dans lequel, contrairement à la télévision, le récit est transmis presque uniquement par les dialogues. La fugacité de la radio, qui n'est pas sans rappeler la tradition orale des récits médiévaux, rend nécessaire l'emploi de ces dialogues dilatés qui, en l'absence de l'image, font office de relais mnémoniques, lesquels, tout comme la répétition, permettent à l'auditeur de ne pas perdre le fil des récits qui se dissipent au fur et à mesure de l'écoute. Si bien que l'absence de sous-texte, la littéralité caractéristique de ce type d'écriture, que l'on serait en droit de reprocher au dramaturge ou à l'auteur de télévision ou de cinéma, se trouve ici justifiée.

Ainsi, la ramification narrative, indissociable d'une sérialité ouverte, c'està-dire le report systématique d'un dénouement définitif, à l'exemple du feuilleton radiophonique et télévisuel américain, le soap opera, constitue la forme privilégiée du radioroman québécois, pour des raisons qui tiennent non seulement à sa diffusion quotidienne mais à sa pérennité (qui peut aller, dans certains cas, jusqu'à plus de 25 ans) et sans doute à l'influence du soap opera lui-même dont certaines productions, à partir de 1938, sont traduites ou adaptées pour la radio québécoise ${ }^{26}$. La version radiophonique du Survenant est éloquente, sur ce point; en effet, contrairement au roman et à la série télévisuelle éponymes, le radioroman ne se termine pas sur le départ définitif (et attendu) de l'étranger: celui-ci reste au Chenal du Moine, empêchant ainsi la tonalité tragique du roman de «survenir ». Le destin d'Angélina, dans le feuilleton, reste suspendu à un dénouement qui se dérobe.

Il vaut la peine qu'on s'attarde aussi au narrateur, car il compte parmi les conventions les plus distinctives du radioroman. Il remplit des fonctions très limitées si on le compare au narrateur du roman-feuilleton, qui est non seulement beaucoup plus présent que le premier, mais aussi plus flamboyant. Cependant, son apport est indispensable dans la mesure où il joue le rôle de "signet auditif ${ }^{27}$ " qui marque les passages d'une intrigue à l'autre, d'un épisode à l'autre. Dans Le Survenant, le narrateur, qui aurait pu, en principe, devenir le véhicule privilégié de plusieurs passages descriptifs et narratifs du roman, ne sert, la plupart du temps, qu'à donner des éléments d'exposition sur l'intrigue, sur le déroulement temporel, les changements de lieu, les transitions entre les scènes ou entre les épisodes. Dans l'extrait de

26 D’après Renée Legris, la programmation québécoise se composait de $25 \%$ de soap operas. Voir Robert Choquette, romancier dramatique de la radio télévision, op. cit., p. 51. Voir son article «Les relations entre la littérature radiophonique du Québec et celle des États-Unis de 1935 à 1945 », Actes du VII ${ }^{e}$ Congrès de l'Association internationale de littérature comparée, Milan V. Dimic et Eva Kushner (dir.), Stuttgart, Kunst und Wissen, coll. «Bibliothèque de la Revue canadienne de littérature comparée», 1979, p. 375-378. 27 L'expression est de moi. 
dialogue cité plus haut, le narrateur établit un pont entre deux scènes, composé d'une synthèse de la scène précédente et de la scène qui va suivre, introduisant ainsi le thème qui va l'animer. À l'occasion, il adopte la forme interrogative, si emblématique du feuilleton littéraire dans son expression du mystère et du suspense:

NARRATEUR Le croirait-on? Le passage au Chenal du Moine des deux belles Soreloises a créé moins d'effet chez les hommes que chez les femmes. Quel crime ont-elles tant commis? Celui d'être belles? Et de vouloir faire la conquête du Survenant?

(RRS, $\mathrm{n}^{\circ} 81,16$ janvier 1963, p. 6)

Ses fonctions sont tout à fait assimilables à celles de la musique puisqu'elles contribuent à lier entre eux les fragments du récit en suggérant atmosphères, émotions et motifs narratifs. Sur ce plan, il faut souligner aussi l'appoint essentiel des effets sonores comme vecteurs sémantiques, tout en restant prudent sur l'efficacité des effets de réel qu'ils pouvaient engendrer, étant donné les limites techniques et financières de la production radiophonique de l'époque. Au-delà de leur utilité en termes de transition d'une scène à l'autre, comme un son de grelots indiquant que les personnages sont à l'extérieur, dans un traineau en marche, les sons traduisent également une foule de mouvements et d'actions, évoquant une gestuelle et une spatialité qui font presque figure d'image sonore: pas sur le plancher ou dans l'escalier, bruits de chaise, de sabots, porte qui s'ouvre ou se ferme, coups de hache, chien qui jappe, cloche d'église, chaudière vide renversée, ces sons évoquent, sur un mode tour à tour trivial et mélancolique, la vie quotidienne d'un temps révolu.

D'autre part, Germaine Guèvremont est très consciente des conditions de production et de la nature particulière des différents médiums, comme le prouvent les multiples didascalies parsemant les scénarii du téléroman Le Survenant. La suggestion suivante, choisie entre mille, l'atteste éloquemment:

\footnotetext{
Le Survenant et Amable scient du bois au godendard (scène plus facile et plus drôle que l'encavement des pommes de terre). Mais les deux hommes ne s'accordent pas. Le Survenant travaille trop vite pour Amable. À tout moment, Amable fait signe d'arrêter. Il s'essuie la figure à l'aide de sa manche de chemise. (TRS, $\mathrm{n}^{\circ} 2$, 9 novembre 1954, p. 8)
}

À l'évidence, l'auteure a compris les ressources de la télévision, son potentiel pour le comique physique, ainsi que la grande quantité d'informations que peut véhiculer l'image. On l'a vu, la paresse d'Amable, dans le radioroman, était commentée; ici, elle est montrée sans que le recours au dialogue ne soit nécessaire. Ceci constitue une différence fondamentale entre le radioroman et le téléroman : même si l'action, pour progresser, s'appuie encore lourdement sur les dialogues, ceux-ci n'expriment plus de façon littérale la pensée des personnages. Un écart se creuse entre ce qui est exprimé tout haut et ce qui se joue sur un plan latent, sous-jacent; en d'autres termes, le sous-texte, nécessairement absent du radioroman, revient en force dans la série télévisée grâce à l'image. À ce chapitre, une foule de détails visuels concourent 
à l'intelligence du récit, à la fois en ce qui concerne la dénotation (ce qu'ils montrent explicitement) et la connotation (ce qu'ils dévoilent du dessous secret des émotions). Ainsi, certains accessoires chargés de sens symbolique dans le roman réapparaissent dans le téléroman: la chaise inoccupée de Mathilde, les plantes en pot et le roman Geneviève de Brabant d'Angélina, la tasse et l'almanach qu'affectionne Phonsine... Mais l'image et la sérialité permettent en outre d'ajouter des leitmotive visuels qui donnent un sens extradiégétique à l'œuvre. La lampe à huile qu'une femme allume soigneusement au début du générique de chaque épisode est très révélatrice à cet égard: emblème de la chaleur de la vie domestique et de la stabilité, elle représente de même un passé qui n'est plus accessible que par la fiction, ce que renforce l'apposition visuelle du roman Le Survenant, déposé juste à côté de la lampe, sur un secrétaire. Une main ouvre le volume sur la page de titre sur laquelle le nom de l'auteure, Germaine Guèvremont, lu par un narrateur, est écrit; puis la main tourne de nouveau la page, nous faisant accéder au premier chapitre. La présence du livre dans un autre médium, écho de l'origine première du récit, ne devrait cependant pas nous leurrer sur sa portée; car s'il confère à la nouvelle œuvre médiatique une forme de légitimité culturelle, il doit tout de même céder sa place à une nouvelle configuration expressive qu'il est impuissant à véhiculer.

Ainsi donc, l'expansion sérielle des romans de Germaine Guèvremont, selon un contexte médiatique différent, a obligé l'auteure à créer des configurations narratives et esthétiques nouvelles qui, tout en possédant des analogies avec l'œuvre romanesque et les traditions littéraires dont elle dérive, s'en éloignent sur d'autres plans. À cet égard, loin d'être mue par un souci mercantile (le préjugé voulant qu'argent rime nécessairement avec dégradation artistique est encore très tenace de nos jours), Guèvremont, on peut le supposer, a choisi de reprendre et de poursuivre l'œuvre déjà établie dans des médias populaires, pressentant que la radio et la télévision, malgré leurs contraintes parfois débilitantes, lui permettraient d'ouvrir son univers fictionnel à une multiplicité de questions contradictoires ancrées dans la modernité. 